\title{
Wheeled Mobile Robot Path Planning and Path Tracking Controller Algorithms: A Review
}

\author{
Oluwaseun. O. Martins ${ }^{1, *}$, Adefemi. A. Adekunle ${ }^{1}$, Samuel. B. Adejuyigbe ${ }^{1}$, Oluwole. H. Adeyemi ${ }^{2}$ and \\ Michael. O. Arowolo ${ }^{1}$
}

\author{
${ }^{1}$ Department of Mechatronics Engineering, Federal University Oye-Ekiti Ekiti State, Nigeria \\ ${ }^{2}$ Department of Agricultural and Mechanical Engineering, Olabisi Onabanjo University, Nigeria
}

Received 18 December 2019; Accepted 21 May 2020

\begin{abstract}
The two major problems in wheeled mobile robot technology is path planning and path tracking. The former evaluates and identifies an obstacle free path for a mobile robot to traverse within its environment and the later deals with the controller design for a mobile robot to track the reference path with precision. Hence, researchers have proposed and applied several solution approaches to these problems over the years. The sustained integration of wheeled mobile robot to task that further require their operation within the human environment characterized with uncertainty makes a review of these solution approaches very significant. Thus, this paper therefore, presents a review of wheeled mobile robot path planning algorithms and path tracking control algorithms applied within the last decennium.
\end{abstract}

Keywords: Path planning, Path tracking, Wheeled mobile robots, Algorithms, Robots

\section{Introduction}

Wheeled mobile robots (WMRs) have become more versatile in their application to various tasks in recent time. The application environments of these robot's ranges from industrial, military, hospitals, schools, offices etc. [1-3]. These environments most often are not precisely engineered for WMRs inclusion. However, with the development of computers and sensory technology, the WMRs industry has seen continual development and received extensive research attention. WMR is a robot that autonomously traverse from a specific start coordinate to a desired goal coordinate within a predefined environment while achieving a specific task [4]. The predefined environment is categorized as static or dynamic and the obstacles within the environment can be static obstacle, dynamic obstacle or a combination of both $[5,6]$. The development of WMRs requires solution to path planning and path tracking problems [6]. Solution to these problems develops intelligence into the robot capable of achieving relation between perception and action [4].

Path planning algorithms evaluate and identifies an obstacle free path for a mobile robot to traverse within its environment [7] and path tracking deals with the controller design for a mobile robot to track the reference path with precision [8]. In literature, path planning algorithms are subcategorized as global and local [7,9]. Global path planning algorithms such as Dijkstra algorithm is applied exclusively to static environment with static obstacles and the robot has comprehensive knowledge of the environment. The path to traverse is generated off-line before the translation of the robot within the environment $[9,10]$. Local path planning

*E-mail address: oluwaseun.martins@fuoye.edu.ng ISSN: $1791-2377 @ 2020$ School of Science, IHU. All rights reserved. doi:10.25103/jestr.133.17 algorithms such as Neural Network [11], Simulated annealing algorithm [12], Near shortest path for Mobile Robot [7] enable the robot to generate a new path in real-time in response to onboard sensory information [13]. This allows the robot traverse safely in clustered environment [4].

Path planning controllers such as hybrid back stepping and adaptive integral sliding mode controller $[6,14,15]$, Proportional Integral Derivative (PID) controller with advance tuning algorithms $[2,16]$ have been applied in the design of appropriate path tracking controller in literature. However, the effective design of these controllers involves the WMR kinematic model and dynamic model [8]. WMR kinematic model presents the constraints between the positions, velocities, and accelerations of the WMR body, wheels and steering links to determine the WMR's linear velocity and angular velocity $[1,4]$. Kinematic model is of forward and inverse relation. Given the WMR individual wheel angular velocity the forward kinematic determines the position and orientation of the WMR and the inverse kinematic does the direct opposite. Dynamic model of WMR predicts it movement. Forward dynamic model defines the WMR response in relation to a given force or torque utilized by the motors and the inverse dynamic model determine the forces or torque to be utilized by the motors to attain the WMR predefined trajectory $[1,4]$.

Therefore, this paper present's a review on WMR path planning and path tracking control algorithms applied within the last decennium. Hence, the following aspects of WMRs are considered: path planning algorithms, path tracking controller design, kinematic modeling, dynamic modeling, model reference adaptive control, sliding-mode control, fuzzy and neural control, vision-based control etc.

The remainder of this paper is organized thus, literature review of path planning algorithms, formulation of kinematic and dynamic model of WMR, literature review of path 
controller algorithms, and finally conclusion of this review paper.

\section{Path Planning Algorithms}

Within the last decennium researchers have approach the path planning problem by modelling the WMR environment and applying a distinctive method to determine the solution. Sheth et al [17] presents a vision-based system for path planning of a WMR within a workspace environment. The overhead camera captures the image of the workspace environment including the start and finish coordinate with obstacle positions in a single frame. The homographic function in Open Computer Vision (OpenCV) is use to process the image, then obstacle characteristic and location is evaluated from the homograph image. The Rapid Exploring Random Tree (RRT) Artificial Intelligence algorithm map out an optimal path accordingly. Although the proposed approach is applicable but the environment must be captured within a single frame of the camera. Culler and Long [18] has design the path planning of a multiply collaborative WMR using vision-based approach. Kinect camera is use for image capturing and OpenCV is use for image processing and onboard sensors for obstacle detection. Ito et at [19] present similar work using small imaging sensor named Single-Photon Avalanche Diode (SPAD) LIDAR. Kala et al [20] address the time complexity problem with Evolutionary Algorithm (EA) for path planning for WMR in dynamic environment using hierarchical EA. Coarser hierarchy EA is responsible for optimization of global path and finer hierarchy EA determines the real-time traverse of the mobile robot on the global path. Simulation of the proposed algorithm was done using a simulator built on JAVA platform. Results exhibits the capability of the WMR to solve complicated maps, avoid static, dynamic and sudden obstacles and successfully attain the goal coordinate.

Tazir et al [21] present a static and dynamic hierarchy path planning algorithm to take advantage of the global static map and local information from onboard sensors. In the static hierarchy, the global optimal path is plan using Genetic Algorithms (GA) with Dijkstra Algorithm (DA) through static obstacles. The local path planning algorithm using DA to generate new path engages when a dynamic obstacle suddenly intercepts the reference path from the static hierarchy path planning algorithm. Computer simulation in Matlab R2010a software shows that the robot attains the target point, in less execution time with optimal path without collision with obstacle in a dynamic complex environment. Toolika et al [22] applied the GA for robot path planning in a dynamic environment. Silva-Ortigoza et al [23] describe the application of artificial potential field for optimal path generation and obstacle avoidance within a workspace environment for a differential drive WMR. Computer simulation in Matlab-Simulink presents the effectiveness of the approach in the generation of obstacle free path for the two wheeled differential drive WMR. Qing [5] presents an improve DA for path planning for WMR in a dynamic environment base on based on the shortest path and travel time optimization criterion. The improve DA stores all equidistant shortest path from start point and goal point during path search. Then considering the number of turns the algorithm identify the optimal path. This is the improvement over the conventional DA. Simulation model was developed with Visual $\mathrm{C}++2010$ and result shows that this algorithm presents the shortest path from equidistant shortest path having considered the number of turns. Zhang and Li [9] design a rapid path planning algorithm with the combination of the Dijkstra's algorithm, A* algorithm, and rolling window principle for mobile robot in dynamic environment. The initial global path is search using the Dijkstra's algorithm. If the possibility of collision with an inbound dynamic obstacle on this reference path is evident, this will activate the rolling window principle. This principle determines a local optimal target state within the detection range of the sensors for the robot to wait. The $\mathrm{A}^{*}$ algorithm then activates to find a new optimal path to the goal point form this target state (current location of the robot). Simulation is use to investigate the efficacy of the algorithm and performance comparison with Ant Colony Optimization (ACO) algorithm, A* algorithm, and $\mathrm{D}^{*}$ algorithm. The result show that the algorithm is not only applicable to dynamic obstacle but also having the least re-routing time of about $99.7 \%$ compared with the other algorithm considered.

Nguyen and Xuan [7] develop and employ the Nearshortest path for Mobile Robot (NSPMR) local path planning algorithm for WMR. The merit of the NSPMR is in twofold; one is that this algorithm considers the shortest path from the start point to the target point and ensures the robot traverse the best moving direction. two, the issue of infinite loop traps of several obstacles in unknown environments is address by the intelligent obstacle avoidance employed. The robot localizes itself, evaluate is traverse direction and distance covered through its onboard Global Positioning System (GPS) and Compass modules. Simulation in Matlab with performance comparison with Bug 1 and Bug 2 algorithms shows with the NSPMR the robot traverses the shortest path. Wu and Feng [24] describe a path planning method for mobile robot within an environment with static and dynamic obstacles based on the combination of static global and the dynamic local path planning methods. The $\mathrm{A}^{*}$ algorithm is use to obtain the static optimal path within the desire coordinates base on initial static obstacle assumption. Then real-time collision avoidance with dynamic obstacles is achieved using trajectory prediction. The artificial potentialfield approach is use for local navigation and the local adjustments to the global optimum path using the local rolling window enables the robot to evade inbound dynamic obstacle. Computer simulation shows the effectiveness of the approach which combines the advantage of global path planning in a static environment with the efficiency of local navigation under a dynamic environment. The algorithm adjusts of the path derived from global path planning using the result obtained from the rolling window during local navigation to avoid dynamic obstacles. Raja and Pugazhenthi [25] illustrates a method of global path planning for a mobile robot within a cluttered environment with static and dynamic obstacles having arbitrary shape, size and location. The choice of shortest path between the desire coordinates is on the bases of shortest Euclidean distance. Simulation result of the algorithm in dynamic obstacles having concave, convex and curved shapes shows it efficiency and effectiveness in terms of shortest path, and minimal execution time compared to vertex heuristics algorithm.

El Khaili [26] address the extensive processing time problem with path planning in dynamic environment using a pictorial approach. Authors presents two algorithms for path search using a visibility graph constructed by sliding on the edges of obstacles. MandaI et al [27] presents an algorithm for mobile robot path planning using attractive and repulsive potentials for goal point and obstacles within the unknown environment respectively. Yun et al [28] presents a WMR navigation method in unknown environment with static and 
dynamic obstacles using Genetic Algorithm (GA) base Dynamic Path Planning Algorithm (DPPA). Authors emphasis is on searching the algorithm that avoids acute shaped obstacles in the environment. The research object is the Team AmigoBotTM Robot and the real-time implementation confirm the practicality and robustness of the proposed algorithm. Li et al [29] combines the kinematics and dynamics equation of an omnidirectional wheel mobile robot and potential field method for it control and navigation in a specific workplace environment. Authors address the local minima problem and the goal non-reachable with obstacles nearby problem peculiar to potential field method by incorporating distance between robot and obstacle in the repulsive potential functions for motion planning. For robust controller performance the model predictive control (MPC) has been incorporated. Qualitative result from simulation data shows effectiveness of the proposed approach for omnidirectional wheel mobile robot's navigation. Sprunk et al (2016) describe a navigation system for omnidirectional robots in industrial environment consisting of distinct modules for mapping, localization, trajectory generation and robot control. Computer simulation and implementation of the navigation system on the KUKA omniRob platform was also presented. Xu et al [30] presents a mobile robot path planning method combining Dubbin's path and Bug algorithm for WMR navigation in unknown static environment considering the kinematical characteristics. Simulation result shows the effectiveness of this method for a WMR to avoid static and dynamic obstacles within the environment. Ayomoh et al [31] describe a path planning mathematical model for a mobile robot in a multi-goal environment comprising of unknown static and dynamic obstacles. However, in recent literature, there has been a paradigm shift from the classical geometric method of environment modelling as a result of excessive computational time and the local minimum problem [32]. Hence, recent literature has been largely on human behavioral and bioinspired approach and Machine Learning for local path planning [33].

\section{Human Behavioral and Bio-inspired Approaches}

Ren et al [34] Developed human like intelligence into WMR operating within a dynamic and unknown environment using fuzzy logic. Hence, the robot understands it environment through perception. Farooq et al [35] described the effectiveness of zero order Takagi-Sugeno and Mamdani-type fuzzy logic controller for mobile robot navigation and obstacle avoidance. Onboard ultrasonic sensor provides sensory data for these controllers to control the linear and angular velocity of the robot wheel actuators. Performance comparison of these controller reveal the superiority of Mamdani-type fuzzy logic controller to zero order TakagiSugeno controller in terms of path smoothness. However, zero order Takagi-Sugeno controller utilizes less memory space for real-time microcontroller implementation. The autonomous navigation of a WMR has been achieved using ATMEGA microcontroller based fuzzy logic controller. Sensory information from onboard sensor provides necessary data for the controller to control the wheel actuators [36].

Abdessemed et al [37] presents a hierarchical fuzzy control design base on the combination of fuzzy rules and stereo vision system for navigating collision free path for indoor mobile robot. Algabri et al [38] attempt the improvement of a WMR navigation performance through membership function parameters optimization of the fuzzy controller. This was achieved by merging fuzzy logic and another optimization algorithm such as Particle Swarm Optimization (PSO). Hmeyda and Bouani [32] has presented a vision-based approach to perceive static obstacle and generate optimal path using PSO for a WMR. External USB Camera is used for image capturing then image processing algorithm extracts the robot position, static obstacle positions and target position. PSO is used to generate optimal path from the robot current position to the target position avoiding static obstacles. Similarly, Mahmud et al [39] similarly presented a vision based Kohonen-type artificial neural network. Ahmadzadeh and Ghanavati, [40] described a navigation method for multiple robot in an environment using a PSO algorithm. The effectiveness of the proposed algorithm is evident in the capability of the robots to navigate in relation to the global best position of a particle in every iteration. Castillo et al [41] in the design of an intelligent controller for a WMR the hybridization of PSO algorithm and ACO algorithm was presented. The hybridized algorithm was used to optimize the membership function of a fuzzy controller. Zhang et al [42] the path planning problem in a dynamic environment has been addressed using a Multi-Objective PSO Algorithm. Shiltagh and Jalal [43] in order to improve the convergence rate of PSO a modified PSO was presented. The effectiveness of the modified PSO was investigated in the searching of shortest path in environment for mobile robot between two defined coordinates avoiding obstacles. Chung et al [44] The autonomous navigation of WMR was achieved using two levels of control. Authors applied PSO algorithm for navigation through obstacles in the environment and fuzzy control for turning angle control.

Juang and Chang [45] presents the possibility of automatic learning of fuzzy logic system through evolutionary-group-based PSO has been presented and applied to WMR navigation in an unknown environment. Allawi and Abdalla [46] have applied PSO algorithm in the determination of optimal parameter of fuzzy type- 2 controller input/output membership function and used for multiple mobile robot navigation. Dongshu et al [47] develop a behavior-based fuzzy logic controller to address the navigation problem of WMR in a dynamic and unknown environment. Their focus with this approach is to develop intelligence in the robot to evade a cul de sac. Similarly, A multi-agent fuzzy logic intelligent control system has been developed by Ayari et al [48] for autonomous navigation of WMR in a dynamic environment. Nichols et al [49] design a wall following WMR using biologically inspired neural network. Al-Jarrah et al [50] proposed a probabilistic neurofuzzy architecture a combination of first order Sugeno fuzzy inference model and adaptive neuro-fuzzy inference system. The possibility of the proposed architecture for achieving multiple WMRs path planning and coordination within a predefined environment was presented. The control of position and orientation of the WMRs follows the model of leader-follower. Kim and Chwa [51] address the problem using a type-2 fuzzy neural network for WMR. The inputs of the proposed methodology are distance form robot to the goal and nearby obstacle, the goal angle and obstacle angle. The output is the controlled linear and angular velocity of the robot to navigate through the environment avoiding the obstacles and reaching the goal. Brahmi et al [52] presents the feasibility of recurrent neural network in the development of intelligent path planning algorithm for autonomous WMR navigating within an undetermined environment. Zhao and Wang [53] addressed the navigation problem of WMR by 
merging sensory information from sonar sensors with neural network. Kumar and Dhama [54] motion and orientation control of WMR within a clustered environment with the combination of fuzzy rule-based and neural network was presented. Pothal and Parhi [55] presents the navigation of multiple WMRs using a sensor based adaptive neuro-fuzzy inference system. The controller enables the robots to navigate through a highly clutter environment avoiding obstacles and reaching the goal successfully.

Mohanta et al [56] the navigation path length optimization for multiple mobile robots has been design using a Petrigenetic algorithm in an unstructured environment. Tuncer and Yildirim [57] compared the performance of the conventional genetic algorithm to a proposed a new mutation operator for a genetic algorithm for mobile robot navigation in a dynamic environment. Computer simulation for the validation shows the superiority of the proposed algorithm. Arora et al [58] describe a genetic algorithm approach to path planning of mobile robot in a dynamic environment. Authors improved on the conventional genetic algorithm by introducing a fitness function based on the Euclidean distance formula between the robot and obstacle. Hussein et al [59] improves the slow convergence rate of the conventional simulated annealing algorithm by combining it with other two metaheuristic optimization algorithms: Tabu Search and genetic algorithm. The design three metaheuristic optimization algorithm is then applied for path planning of mobile robot. Zhang et al [60] proposed the more efficient navigation speed of a mobile robot using a combination of simulated annealing and Ant Colony Optimization (ACO). Synodinos and Aspragathos [61] addressed the local minima problem of mobile robot during traverse with the combination of simulated annealing algorithm and artificial potential field method. Algabri et al [62] presents a differential drive WMR navigation and obstacle avoidance within an unknown environment using an adaptive neuro-fuzzy technique inference system. The validity of the design was done using computer simulation in the Khepera Simulator environment. Singha et al [63] illustrates the efficacy of a biologically inspired neural network (BNN), considering dual weight calculation with an obstacle detection sensor for WMR path planning and mapping in an unknown environment. Computer simulation for validity testing that the target weight algorithm generates less turns and a smaller number of grid cell traversed compared with the local weight algorithm. Hossain and Ferdousand [64] present an optimization technique for autonomous robot path planning in dynamic environment with dynamic obstacles based on Bacterial Foraging Optimization (BFO) method. Liang et al [65] describe the development of a bio-inspired path planning algorithm based on an Adaptive Bacterial Foraging Algorithm (ABFA) for mobile robot. Patle et al [13] presents a real-time navigation algorithm for WMR using a modified Firefly Algorithm (FA) which is studied over Normal Probability Distribution (NPD) in a static clustered environment. Computer simulation and real-world study of the algorithm is done with the Khepera robot programmed using $\mathrm{C}++$ for path planning in real time environment amidst static obstacles. Result shows the effectiveness of their algorithm to find an optimal path for WMR amidst regular shaped static obstacles. Liang and Lee [66] design a local path planning algorithm for multiple WMR application heading for same goal point within a workspace environment using Efficient Artificial Bee Colony (EABC) algorithm. The EABC considered the hybrid objective functions for distances between target, other mobile robots, and obstacles for real-time path planning of each mobile robot. Computer simulation and comparison of the EABC with $\mathrm{ABC}$ shows that with the EABC presents accurate performance than the conventional ABC. However, the computational time of the $\mathrm{EABC}$ was expanded due to the adoption of multiple strategies.

Brand and $\mathrm{Yu}$ [67] presents the comparison of the FA with ACO algorithm in the determination of optimal path for a mobile robot in a 2D static and dynamic environment. Their result shows the superiority of FA to ACO algorithm in the considered performance metric: path length and computational cost. Mohajer et al [68] presents an optimization algorithm inspired by BFA for local path planning of mobile robot. The proposed optimization algorithm: Random Particle Optimization Algorithm randomly search optimal path in a dynamic and unknown environment with dynamic obstacles using onboard sensory information's. Purian and Sadeghian [69] presents WMR path planning using ACO and fuzzy logic algorithm in an unknown dynamic environment. The optimal value from the fuzzy rule table is searched using the ACO thus, minimizing the path length from start coordinate to goal coordinate. Ganapathy et al [70] an improved ACO was presented and evaluated based on three behaviors: goal seeking, wall-following and obstacle avoidance for WMR navigation optimization. Hsu et al [71] presents an improve ACO for WMRs path planning by incorporating a phenomenal updating parameter. Ganganath et al [73] presents a local path planning algorithm for a nonholonomic WMR using ACO algorithm. Juang et al [73] presents the optimal navigation of two mobile robots cooperatively carrying object in an unknown environment using fuzzy controllers, continuous ACO and PSO. Fuzzy controllers are applied for navigation and continuous ACO and PSO for obstacle boundary following objective. Other representative references with differs complexities and applications are in [74-79]

\section{Machine Learning Approach}

The Machine Learning approach applied in robotics path planning is Reinforcement Learning (RL). Similar to how humans discover their environment through continual interaction, $\mathrm{RL}$ is the competence of an autonomous agent to learn and improve it behavior base on it experience in its environment [80]. The agent (WMR) through RL autonomously uncover an optimal behavior through trial-anderror interactions with its environment. RL is premise on reward and sanction. For every good behavior the agent gets a scalar reward or a scalar sanction if otherwise. Therefore, an agent primary objective is to maximize the accrued reward over its lifetime. Thus, it's an associative study between environment states and actions [81, 82]. Fig. 1 illustrates the principle of RL.

The value functions in RL; shortest path, the path with the shortest time, the safest path, or any combination of different sub-objectives are to be maximized through the action policy to attain a predefine goal state. Exploration and Exploitation are referred to as the control strategy in RL. Exploration is when an agent takes an action with nonzero probability in every found state to learn the environment. However, in exploitation the agent adopts exclusively it present knowledge in anticipation of good performance by selecting greedy actions [84]. Thus, the goal is for the agent to achieve a balance between Exploration/Exploitation through some strategy such as $\varepsilon$-greedy exploration, Boltzmann exploration, Optimistic Initial Value (OIV), Extreme learning machine 
(ELM) etc. [82, 83, 85, 86]. The Q-Learning algorithm develop by Watkins is one of the most frequently used technique in solving RL problems [87]. It mathematical model is premise on the Markov chain and dynamic programming in combination with the knowledge of animal behavioral psychology, to attain an agent online learning [83, 85].

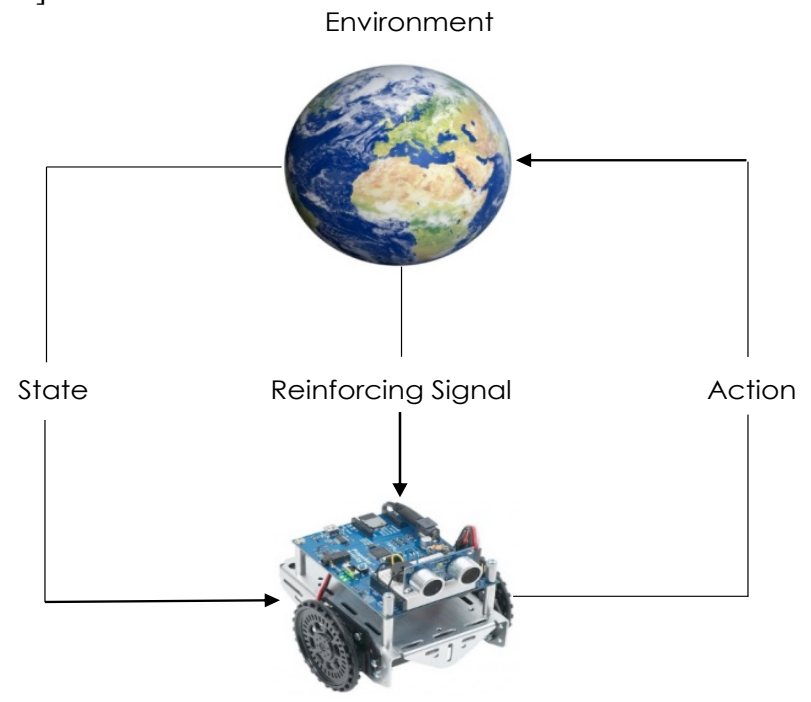

Agent (Mobile Robot)

Fig. 1. Principle of RL

Xiaoyun et al [33] presents the combination of $\varepsilon$-greedy (epsilon-greedy) and Boltzmann exploration strategy in a modified Q-learning algorithm for selecting an action at the current state for learning agent navigation policies in a modelfree manner. The aim of merging this exploration strategy is to avoid local minimum and stimulate the convergence rate. Computer simulation in Matlab environment, under constant reward matrix and a varying reward matrix with probabilistic outcomes was considered. The value function is the shortest path with reward maximization. Qualitative results show that a discount factor $(\gamma) \leq 0.9$ achieved the shortest path with maximum accumulated reward. Similarly, at $\gamma \leq 0.9$ the modified Q-learning converged faster compared with the conventional Q-learning (that only uses $\varepsilon$-greedy exploration) which is yet to converge at the end of the training episodes. Ribeiro et al [86] describe the application of two distinct Qlearning algorithms for path planning of an agent premise on the $\varepsilon$-greedy exploration strategy. The distinctive feature of these algorithm is that; one allows the agent to take an action at predefined interval even when the state space signal is constant and the other permit an action of the agent only when the state space signal changes. Simulation results shows that the latter approach out performs the former in terms of learning quickness and robustness.

Watchanupaporn and Pudtuan [88] attempts to increase the learning rate of a group of WMR heading for the same goal point by combining Q-learning algorithm with PSO within a simulated grid environment. Authors modified the conventional Q-learning algorithm for multi-robot problem and combined this algorithm with the PSO algorithm. The training of algorithm adopts four methods: Q-learning (QL), modified Q-learning (mQL), QL+PSO, and mQL+PSO. This algorithm was trained using 5, 10, 15, 20 and 25 robot per group respectively and performance compared accordingly. Simulation of this approach in three different environments with diverse level of difficulty shows that that learning rate increases with number of robots. Learning performance of the group was improved when QL and $\mathrm{mQL}$ was combined with PSO. Also, as difficulty level increase QL tend to slowdown as the number of robots increases however, mQL still learns faster even with increased number of robots. Ren et al [83] describe the feasibility of Q-learning base on extreme learning machine (Q-ELM algorithm) in addressing mobile robot path planning problems. These problems include; high dimensionality, training difficulty and slow learning speed associated with the application of behavioral psychology neural network in mobile robot path planning. authors substantiate the feasibility of the Q-ELM through experiments carried out by Matlab 7.0. Results shows that the stability and convergence of the algorithm are proved by number of episodes. The advantage of ELM in terms of fast computing power, inherently accelerates the implementation of Qlearning in the agent and improves the learning rate. Valiollahi et al [89] describe the combination of fuzzy logic and Q-learning algorithm for autonomous navigations of a Khepera mobile robot. Sensory data by limited range infrared sensors is the Fuzzy inputs. The fuzzy outputs are the robot speed and steering angle. Every sensed data is fuzzified for efficient handling of uncertain, imprecise, or noisy information. Q-learning algorithm is used for the online tuning of the fuzzy inference. This provides a flexible decision-making system, with inherent adaptability to unknown environments. Experiment carried out on 30 simulated environments reveals goal reaching probability is $95 \%$ and the tendency of the robot to go beyond the safe margin from obstacles or walls is $15 \%$. Xu et al [90] attempts the reduction of computation time and memory space of Qlearning algorithm. They simplified the algorithm discrete value table into a new version that stores only one optimum action and its Q-value rather than storing every action's Qvalue in each state for conventional Q-learning. Authors implemented their simplified online Q-learning (SOQ) algorithm in Java language using the LeJOS implementation for the Lego Mindstorms EV3 robot. Experimental result shows the SOQ algorithm promising capability of reduced memory complexity when dealing with thousands of stateaction values. Zheng et al [91] describe a solution to autonomous obstacle avoidance problem of mobile robot in static and dynamic environment. They applied the feature level fusion to fuse the sensory information of laser sensor and sonar sensor to complement their single application drawbacks. Q-learning algorithm is then applied to avoid dynamic obstacles as the robot heads for the goal coordinate. Their experimental setup is divided into; simulation in Matlab and actual mobile robot experiment. Experimental result shows that the robot can navigate through obstacles and safely attain the goal coordinate.

Muhammad and Bucak [87] attempt to solve the rate of convergence of the Q-table in large state-action space associated with mobile robot application. The Q-table stores the state-action pair. Their approach in solving this problem is to store all the state-action values visited by the mobile robot from start coordinate to the goal coordinate and then replaying or backtracking these state-action sequences to use the updated $\mathrm{Q}$ values after the goal state has been reached. The exploration/exploitation strategy of action-selection mechanism is the Boltzman or soft-max distribution. Simulation of this approach and it comparison with the conventional Q-learning algorithm in terms of cumulative change in Q-value and rate of change of the Q values was presented. The result shows significant improvement in the rate of change of the Q-table by the proposed algorithm as compared to the conventional Q-learning. Yang and Li [85] 
describe the navigation control algorithm of a mobile robot base on Q-learning method. The authors considered only obstacles within 180 o range ahead of the robot for algorithm simplicity and obstacles position is divided into the left, right and ahead directions. The Boltzmann selection mechanism is adopted for the training process to action selection. The feasibility of this algorithm is verified by two different simulation environments setups. Results after 237 training episodes presents a feasible path compared and robot attains the goal coordinate safely. Lakhmi and Atulya [82] propose a modification of the conventional Q-learning (CQL) algorithm and evaluate it increase performance in the path-planning problem. Their approach consists of four conditional rules for balancing the exploration/ exploitation factor. The feasibility of the proposed algorithm is evaluated using computer simulation and a created real-world environment. In the computer simulation a grid map of $20 \times 20$ under four experimental settings were used to compare the performance of the MQL, EQL and CQL. result shows MQL outperforms the CQL and EQL in all cases. Sichkar [92] evaluates the performance of Q-Learning algorithm and its modification SARSA reinforcement algorithm for global path planning for mobile robots. Cumulatively, 50 experiment were conducted for Q-Learning and SARSA algorithm with different parameters. The results show that Boltzmann distribution temperature parameter $\mathrm{T}$ from 0.01 to 0.04 for 1000 episodes achieved optimal solution between quality and learning speed.

Jiang and Xin [93] describe a novel learning algorithm for path planning of mobile robots in a large state space environment. Fuzzy rule is applied to fraction the state and action. Their approach is critical in large space application with Q-learning algorithm where the number of states and the lengths and directions of the actions are infinite. To achieve a trade-off between exploration and exploitation authors combine the $\varepsilon$-greedy with a described area allocation strategy to improve the learning convergence speed. Experimental set up was applied to evaluate the feasibility of the describe approach. The superiority of the proposed algorithm over the $\varepsilon$-greedy method and the SoftMax is evident. Li et al [94] describe an innovative path planning method based on improved Q-Learning algorithm (IQL) and some heuristic searching strategies for mobile robot path planning problem in dynamic environment. The IQL combines the $\varepsilon$-greedy exploration with Boltzmann exploration to attain the balance between the exploration and exploitation appropriately. The heuristic searching strategies shows it significance in reducing the number of iterations in learning process and controlling the variation range of robot orientation angle. Authors validate their IQL approach via experimental set up and compare it performance with the performance of the IQL was compared with CQL, A* and EQL algorithm. Qualitative result shows IQL superiority to CQL in both distance and orientation angle of the path. Although the $A^{*}$ presents shorter distance but it doesn't take the safe range with the obstacle nearby. Motlagh et al [95] design obstacle avoidance and goal seeking competence in a mobile robot with the combination of reinforcement learning and neural networks. Roy et al [96] presents the combination of image processing and Q-learning to solve the path planning problem of WMR in an indoor environment. The environment image is captured using a ceiling mounted camera. This image processed and the obstacles in the environment is processed using Adaptive Gaussian Threshold. Trajectory tracking for the robot is done using OpenCV template matching.

\section{Path Tracking Algorithms}

The efficient controller design for a differential drive WMR requires the incorporation of it kinematic and dynamic model. The general schematic of a differential drive WMR is given in Fig. 2.

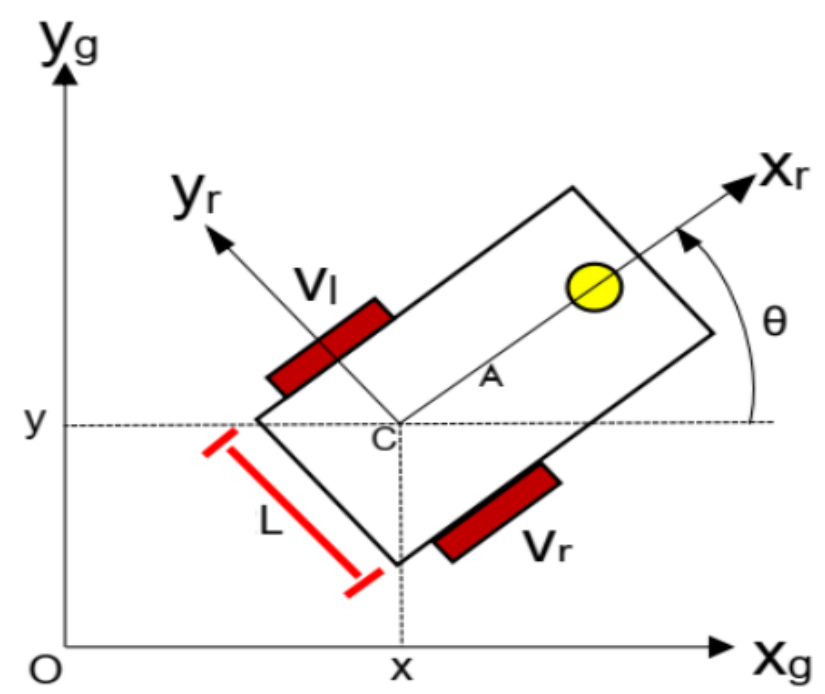

Fig. 2 General Schematic of a Differential Drive WMR

where $\left\{x_{g} O y_{g}\right\}$ represents the global reference frame and $\left\{x_{r} C y_{r}\right\}$ represents the mobile robots local reference frame. The left and right wheel velocity is $v_{l}$ and $v_{r}$ respectively. The position of the center of the robot wheel at $C$ relative to the global reference frame is located by coordinate $x$ and $y . \varphi$ is the difference in orientation between the global and local reference frames. The kinematic model of the mobile robot as described by $[1,8,97]$ is given below:

$$
\left\{\begin{array}{c}
x=\frac{r}{2}\left(v_{r}+v_{l}\right) \cos \phi \\
y=\frac{r}{2}\left(v_{r}+v_{l}\right) \sin \phi \\
\dot{\phi}=\frac{r}{L}\left(v_{r}-v_{l}\right)
\end{array}\right.
$$

Eq.1 presents the differential drive wheeled mobile robot kinematic model. Showing how $v_{l}$ and $v_{r}$ transforms to $\dot{x}, \dot{y}$ and $\dot{\varphi}$. Where $r$ is the wheel radius, $L$ is the distance between the wheels; and $L / 2$ is distance of individual wheel to the center $C$ of the mobile robot. However, is it more convenient to describe the linear velocity and rotation velocity of a WMR using single variable $v_{\text {robot }}$ and $\omega_{\text {robot }}$ rather than $v_{l}$ and $v_{r}$ as in Eq.1. Hence, the kinematic model of a differential drive WMR is mapped with that of its unicycle WMR counterpart $[8,97]$. The kinematic model of a unicycle is 


$$
\left\{\begin{array}{c}
x=v \cos \phi \\
y=v \sin \phi \\
\dot{\phi}=\omega
\end{array}\right.
$$

Comparing Eq. 1 and Eq.2

$$
\begin{aligned}
& V_{\text {robot }}=\frac{r}{2}\left(v_{r}+v_{l}\right) \\
& \omega_{\text {robot }}=\frac{r}{L}\left(v_{r}-v_{l}\right)
\end{aligned}
$$

So, Eq.3 and Eq.4 establish the connection between the translational velocity $v$ of the robot to wheel velocities $v_{l}$ and $v_{r}$ and angular velocity $\omega$ of the robot to wheel velocities $v_{l}$ and $v_{r}$ respectively. Solving Eq. 3 and 4 simultaneously we obtain Eq. 5 and 6 for $v_{r}$ and $v_{l}$ respectively:

$$
\begin{aligned}
& v_{r}=\frac{2 V_{\text {robot }}+\omega_{\text {robot }} L}{2 r} \\
& v_{l}=\frac{2 V_{\text {robot }}-\omega_{\text {robot }} L}{2 r}
\end{aligned}
$$

Hence, from Eq. 5 and 6 we have $v_{l}$ and $v_{r}$ in terms of the design parameter $v$ and $\omega$ and the measured parameter $L$ and $r$.

Similarly, the relation between the wheel velocities $v_{l}, v_{r}$ and the robot velocity $v$ and angular velocity $\omega$ as given by $[1,8]$ :

$$
\left\{\begin{array}{l}
\omega_{\text {robot }}=\frac{\left(v_{r}-v_{l}\right)}{L} \\
V_{\text {robot }}=\frac{\left(v_{r}+v_{l}\right)}{2}
\end{array}\right.
$$

It is important to state that the wheel velocities $v_{l}, v_{r}$ is derived from the DC motor velocities $\omega_{l}$ and $\omega_{r}$ with the relations $[1,8]$ :

$$
\left\{\begin{array}{l}
v_{r}=r \omega_{r} \\
v_{l}=r \omega_{l}
\end{array}\right.
$$

Inserting Eq.8 into Eq.7, Eq.9 is obtained:

$$
\begin{aligned}
& \left\{\begin{array}{l}
\omega_{\text {robot }}=\frac{\left(r \omega_{r}-r \omega_{l}\right)}{L} \\
V_{\text {robot }}=\frac{\left(r \omega_{r}+r \omega_{l}\right)}{2}
\end{array}\right. \\
& \left\{\begin{array}{l}
\omega_{\text {robot }}=\frac{r}{L}\left(\omega_{r}-\omega_{l}\right) \\
V_{\text {robot }}=\frac{r}{2}\left(\omega_{r}+\omega_{l}\right)
\end{array}\right.
\end{aligned}
$$

So, Eq.10 presents the relation of robot translational velocity and rotational velocity to DC motor velocity.

The Dynamic model of a differential drive WMR can be derived from the Euler - Lagrange formulation with the general form $[1,8,114]$ :

$\frac{d}{d t}\left(\frac{\partial L}{\partial \dot{q}_{i}}\right)-\frac{\partial L}{\partial \dot{q}_{i}}=Q_{i}$

Where, $L$ is the Lagrange function, expressed as the difference between the WMR Kinetic Energy (K.E) and WMR Potential Energy (P.E)? However, the P.E is set as zero since robot is required to traverse on a plane. Hence, $L=$ K. $E(K)$.

$\frac{d}{d t}\left(\frac{\partial K}{\partial \dot{q_{i}}}\right)-\frac{\partial K}{\partial \dot{q_{i}}}=Q_{i}$

The summation of K.E on the WMR is:

$K=K_{t}+K_{r}+K_{w r}$

$q_{i}$ is the WMR global coordinate

$Q_{i}$ is the global force acting on the WMR?

$K_{t}$ is the K.E associated with WMR translation

$K_{r}$ is the K.E due to WMR rotation

$K_{w r}$ is the K.E due to WMR wheel and rotor rotations.

The nonlinear nature of the WMR system makes the need for a controller inevitable. Thus, controller design have been adopted in literature to compensate for the system uncertainty and input disturbance. Hmeyda and Bouani [32] has presented a dual PID controller to output the linear velocity and angular velocity of a WMR wheel motor. The reference path is generated using PSO-Trajectory algorithm. However, the kinematic and dynamic model of the WMR was not incorporated and advance tuning algorithms for PID gains was not considered. Hence limiting the robustness of these approach to static environement with static obstacles. Rai and Rai [98] describe the speed control for an Arduino Uno microcontroller-based DC motor. They merge a multilayer neural network controller and PID controller to improve the performance of the controller. Rossomando and Soria [99] address the trajectory tracking control problem of a WMR using their designed adaptive neural network PID controller. Al Mutib and Mattar [100] have design ta differential drive WMR wheel actuator speed control using neuro-fuzzy controller. The input to the controller is the sensory information from onboard ultrasonic sensor for obstacle detection. Baturone et al [101] describe a car-like mobile robot control using an embedded neuro-fuzzy controller.

Deshpande and Bhosale [102] introduce the adaptive neuro-fuzzy inference system controller for navigation control of a differential drive WMR with nonholonomic constraints. Al-Mayyahi et al [103] describe the control of a differential drive WMR wheel actuator angular velocity and heading angle control using an adaptive neuro-fuzzy technique inference system controller. Silva-Ortigoza et al [23] address the path tracking problem considering the 


\section{Journal of Engineering Science and Technology Review 13 (3) (2020) 152 - 164}

kinematic model of a two wheel differential drive WMR using Hierarchical control. The desired velocity profiles of the two wheels are generated using the upper level, input-output linearization control for the movement of the WMR along the reference path avoiding obstacles. A dual PI controller is use to control the DC motor of the right and left actuated wheel velocity respectively at the lower level. The lower level control is responsible for synchronizing the desire velocity obtain at the upper level with the actual velocity of the robot wheel hence, tracking the path. Simulation result shows some deviation between the actual velocities and the desire velocities using the PI controller. This indicate the necessity for an advance optimization algorithm for tuning the PI gains for better performance. Jayaprakash et al [104] presents sensor fusion method for WMR absolute heading angle detection in an indoor environment. Onboard sensors: Gyroscope (L3G400), encoders and ultrasonic sensors were deployed to measure angular velocity, speed/direction of wheel rotaion and obstacle detection respectively. Kalman filter is applied to fuse the data from the gyroscope and encoders to accurately estimate the real-time heading angle of the WMR. PID controller is use to compensate for the deviation of the real-time heading angle and desire heading angle. Simulation result shows that using Kalman filter and PID controller effectively correct the heading angle deviation to marginal tolerable value of about \pm 2 degrees. Lee and Chia [105] design a straight path control of a four omnidirectional WMR wheel actuator DC motors using wheel eoncoder and Proportional controller. The actual wheel velocity is sense through the wheel encoder and sends the data to the onboard personal computer via serial communication using a USB cable. To track straight path deviation from the encoder reading for the right and left motor must tend to zero, thus the proportional controller compensate for this difference in encoder readings. Comaparison of the proposed design with logic control system result shows the proportional control system performed better with minimal oscillation. Koubaà et al [106] address the trajectory tracking problem of WMR with unknown skidding and slipping (dynamic model disturbance) using an adaptive sliding mode control. To eliminate the effect of dynamic model disturbance on the actual velocity resulting in its deviation from the desired velocity, the adaptive sliding-mode dynamic controller is proposed. The confirmation of system stability and the convergence of the tracking errors to zero is done using the Lyapunov theory. Illustration of the effectiveness of the proposed controller through computer simulation presents it robustness and efficiency superior to kinematic/torque controller.

Arantes and Sena Esteves [107] descirbe the wheel actuator velocity control for a four Mecanum wheels omnidirectional mobile robot using PID controller. Susan et al [108] presents fuzzy hybrid PID controller for steering control for an omnidirectional mobile robot with threewheels. The proposed controller is implemented on an autonomous wheel chair with active caster wheels to improve it maneuverability and environment accessibility. Ren et al [109] achieve the friction compensation of a three-wheeled omnidirectional mobile robot using a reduced-order extended state observer (ESO) based sliding mode control scheme. The comparative advantage of the proposed approach is that; precise friction model is unnecessary resulting in minimal computational cost. Mousavi [110] presents a path tracking controller for a mobile robot in a deterministic environment using fuzzy logic. The input variables are distance and angular difference and the two control inputs are linear and angular velocities. Simulation illustration of the proposed method and comparison with model predictive controller (MPC) shows it superiority in terms of speed, robustness and simplicity. Mahgoub and Sanhoury [111] develop a path tracking controller model for a WMR using backstepping approach. The controller development considers the kinematic model of a WMR and the system stability is confirm using Lyapunov function. Alouache and $\mathrm{Wu}$ [112] applied the GA as advance optimization algorithm to improve the peroformance of a PID controller in terms of control precision and speed of convergence for a mobile robot. Investigation of the effectiveness of the proposed GA-PID controller in comparison to PID in tracking a reference trajectory presents it superiority and robustness. Esmaeili et al [113] address the balancing and trajectory tracking problem of Two Wheeled Balancing Mobile Robots using backstepping Sliding Mode Controller (SMC). Lagrangian method with dynamics of DC motors is use to derive the mathematical model of the robot. Mallem et al [114] presents a path tracking method base on PID fast terminal sliding mode dynamic inverse control for WMR. The method considers the kinematics and dynamics models of WMR to ensure the asymptotic stabilization of the robot's position and orientation around the desired trajectory. Simulation results shows the practicability of the proposed method in real-world mobile robot application. Allagui et al [115] presents the trajectory tracking of khepera II WMR using three fuzzy logic PI controllers. The fuzzy logic output is the gains of the PI controller, thus, the quality of trajectory tracking and navigation is improved. Simulation result of the fuzzy PI controller and comparison with PI controller shows the proposed fuzzy PI controller eliminates the sensitivity problem associated with PI controllers. Nikranjbar et al [81] design a path tracking controller for a three-wheel mobile robot in the presence of varying-size triangular regularly shape dynamic obstacle using an an hybrid back stepping kinematic control along with the repressor based adaptive integral sliding mode. This presents the kinematic and dynamic control speedof the robot respectively. Simulation shows significant input disturbance suppression characteristic.

Alakshendra et al [15] evaluates the preformance of the Integral sliding mode controller (ISMC) and adaptive integral sliding mode controller (AISMC) for tracking a U- path on a trio-wheeled omnidirectional mobile robot considering friction disturbance and bounded uncertainties. Results presents the superiority of the AISMC to the ISMC in tracking the desired path. Alakshendra and Chiddarwar [14] discuss the nonlinear trajectory tracking competence of a 4-Mecanum Wheeled Mobile Robot (4-MWMR) using second order sliding mode controller (SSMC). Authors incorporates the equation of motion derive using Newton Euler formulation with regard to motor dynamics, external forces and uncertainties which may vary the mobile robot from its trajectory. Simulation result showed a significant achievement of their proposed controller in ensuring that the 4-MWMR tracks a non-linear reference trajectory successfully. Urrea and Muñoz [116] appraise and reports the perofromance of adaptive PID (ADP PID), model reference adaptive controller (MRAC), and fuzzy controller (FC) employed on a model two Wheeled Mobile Robot for autonomous path tracking on a model farm. The performance metric is the path trajectory generated by the controller and the torque requirement by the actuating motors. Qualitative results of the applied indices shows MRAC presents better result on both metric. Vinod Raj and Abraham [8] address the response delay problem of controller to disturbances using a 
cascaded PID control for reference path tracking considering the kinematic and dynamic model of the robot. This approach adopts the master and slave controller to control the linear and angular velocity of the robot base on it kinematic and dynamic model respectively. The two controllers are base on PID architecture. Simulation conducted for straight and circular path tracking reveals of the controllers ability in tracking the desired path but with visible deviation. This can be improve by using advance algorithms in tuning the PID gains. Yousfi Allagui et al [117] describe the application of three fuzzy logic PI controllers for the purpose of goal reaching and trajectory tracking of the khepera II mobile robot basd on it kinematic model. Individual controller consists of designing a classical PI controller and a fuzzy inference system with two inputs and two outputs. The inputs are the error and the error derivative; however the outputs of the fuzzy unit are the parameter corrections of the gains of the PI controller. The approach is base on conversion of linguistic inference systems into automatic control. The simulation of the approach reveal the desire target and trajectory tracking are realized. The comparison of fuzzy PI compare to classical PI gives satisfactory results due to it elimination of sensitive to initial conditions.

Dwivedi et al [118] presents an hybrid controller comprising of PSO base PID controller and Support Vector Machine (SVM) a supervised machine learning classification algorithm. The PSO base PID controls the wheel velocity and the SVM controls the turning angles by classifying the angles of the next position. The aim of the Authors is to develop a method to find the global optimum value of PID parameters under constraints with simplicity and computationally efficiency. Simulation result presents the robustness and accuracy of the controller in tracking random and circular path. Lee et al [119] presents the Taguchi method to determine the optimal design of PID controller parameters in path tracking task. Authors design a step response tracking task, and test the parameters of PID controller. The Tugachi proofs to be fast and efficient in finding the best combination of PID gain parameters. The tuning time is also minimal compare with manual gains parameter tuning. Song et al [120] presents a control strategy combine with fuzzy control base on the Line of Sight (LOS) method. The research object is the Pioneer-3 wheeled mobile robot. The kinematic model of the mobile robot is developed and the path tracking control performance is evaluated on the straight and circular path. Simulation result reveals success of the propose approach in tracking the straight and circular path with good performance. Shijin and Udayakumar [1] attempts the point tracking control problem of differential drive WMR using a PID controller. Authors describe the speed controller of the DC motors for a differential drive WMR using a PID controller considering it kinematic model and dynamic model. The Ziegler-Nichols method is use to evaluated the PID gains. Qualitative ressult shows the significance of the controller in ensuring the speed set point of $100 \mathrm{rads} / \mathrm{sec}$ is achieved by both wheels. However, on point - to - point tracking the qualitative result shows the mobile robot took a curved path rather than the required straight path. Thus, the need for optimaization algorithm such as PSO and GA to optimize the PID gains for quick response of the controller. Heikkinen et al [121] propose a self-tuning-parameter fuzzy PID controller for rotational speed control of a differential drive WMR DC motors. The aim is to complete a straight trajectory using the self-tuning-parameter fuzzy PID controller on the DC motors. Qualitative result shows the effecitiveness of their approach in tracking a straight path in a wide parameter variation range compare with the conventional PID controller. Meng et al [2] investigates the effectiveness of PID controller as speed controller on a two wheeled differential drive mobile robot for straight and curved path. Their research object is a constructed two-wheel mobile robot platform based on STM32 Micro-controller. Simulation was conducted in Matlab-Simulink for the straight and curved path trajectory to validate the feasibility of the PID controller. resuts reveals centimeter deviations from the desired path sue to lack of robustness of the PID gain parameters.

Zhi et al [122] presents a shift control base on Neural Network and Fuzzy PID as the DC motor speed controller for a differential drive wheeled mobile robot. These advance algorithm for tuning of the PID gains is aimed to offset the limitation of conventional PID for the target purpose. Simulation and effect analysis of the propose method was done in Simulink and the control object is the DC servo motor. The result of the experiment compares the performance of the Fuzzy PID, Neural Network PID and the Neural Network and Fuzzy PID in terms of response time and maximum overshoot (\%) of the set point. The Fuzzy PID control show fast response but with more overshoot converse to the performance of the Neural Network PID control. However, with the Neural Network and Fuzzy PID the demerits both are eliminated and their merits established. Chang and Jin [123] decribe the implementation of an adaptive tracking controller base on the PID for mobile robot trajectory tracking. Their approach incorporates the nonlinear model of the differenetial drive WMR kinematics to ensure a precise prediction of the future trajectories. Qualitative result shows that their propose method has less error and less maximum overshoot than conventional backstepping method and ordinary PID. Ammar and Azar [124] investigates the application of PID controller and Fractional Order PID (FOPID) controller to achieve a robust controller for a differential drive WMR. The research object is the Pioneer-3 Mobile Robot and the control variable is the linear velocity and angular velocity through the linear velocity control loop and angular velocity control loop. Authors apply the Integral square error (ISE), Integral absolute error (IAE), Integral time-square error (ITSE) and Integral time- absolute error (ITAE) tuning algorithms to tune the gains of PID and FOPID. Computer simulation done in Matlab-Simulink and the measured performance metrics are response time, peak time, maximum overshot (\%) and settling time. The result shows that PID-ITAE and FOPID-ISE presents the best parameters for the metric and FOPID-ISE achieved better performance parameter in relation to PID-ITAE. Other representative works are: Tamila et al [125], Salem [126], Barthelmes and Zehnter [127], Proaño et al [128].

\section{Conclusion}

This literature review presents the necessity for path planning and path tracking competence in WMR for it sustained integration to task that further require them to operate within the human environment characterized with uncertainty. Although numerous path planning and path tracking algorithms have been applied by researchers, however, static environment with static obstacles consideration are in the majority. Furthermore, performance evaluation of these algorithms is largely base on computer simulation but some researchers equally describe their algorithm feasibility on real-world robot miniature applications. This creates a gap 
between available methodologies and their real-world practicability. Hence, this gap motivates the current research on the application of Q-learning algorithm for path planning and PID controller for path tracking with advance optimization algorithm in the development of a unit-load dispatch differential drive WMR in an office environment with static and dynamic obstacles. This present papers' emphasis is on review of WMR path planning and path tracking control algorithms. Hence, extensive insight on the discussed technology can be found in the sited references.

This is an Open Access article distributed under the terms of the Creative Commons Attribution License

\section{References}

[1.] Shijin, C., \& Udayakumar, K. (2017). Speed Control of Wheeled Mobile Robots using PID with Dynamic and Kinematic Modelling. International Conference on Innovations in information Embedded and Communication Systems (ICIIECS) (pp. 1-7). IEEE.

[2.] Meng, J., Liu, A., Yang, Y., Wu, Z., \& Xu, Q. (2018). Two-wheeled robot platform based on PID control. 5th International Conference on Information Science and Control Engineering (pp. 1011-1014). IEEE.

[3.] Martins, O. O., Adekunle, A. A., Adejuyigbe, S. B., Arowolo, M. O., \& Adeyemi, H. O. (2019). Prototype Line Following Automatic Guided Vehicle (AGV) For Unit Load Dispatch In an Office Environment. FUDMA Journal of Sciences (FJS) Vol. 3 No. 3, 415 -423 .

[4.] Tzafestas, S. (2018). Mobile Robot Control and Navigation: A Global Overview. Journal of Intelligent \& Robotic Systems, 1-24.

[5.] Qing, G., Zhang, Z., \& Yue, X. (2017). Path-planning of Automated Guided Vehicle based on Improved Dijkstra Algorithm. 29th Chinese Control and Decision Conference (CCDC) (pp. 71387143). IEEE.

[6.] Nikranjbar, A., Haidari, M., \& Atai, A. A. (2018). Adaptive Sliding Mode Tracking Control of Mobile Robot in Dynamic Environment Using Artificial Potential Fields. Journal of Computer \& Robotics, $1-2$.

[7.] Nguyen, H. T., \& Xuan, H. L. (2016). Path planning and Obstacle avoidance approaches for Mobile robot. International Journal of Computer Science Issues, 3-4.

[8.] Vinod Raj, N., \& Abraham, T. M. (2015). Design, Simulation and Implementation of Cascaded Path Tracking Controller for a Differential Drive Mobile Robot. International Conference on Advances in Computing, Communications and Informatics (ICACCI) (pp. 1085-1090). IEEE.

[9.] Zhang, H.-m., \& Li, M.-1. (2017). Rapid path planning algorithm for mobile robot in dynamic environment. Advances in Mechanical Engineering, 1-12. doi: doi:10.1177/1687814017747400

[10.] Shamsinejad, P., Saraee, M., \& Sheikholeslam, F. (2010). A new path planner for autonomous mobile robots based on genetic algorithm. 3rd International Conference on Computer Science and Information Technology, (pp. 115-116).

[11.] Engedy, I., \& Horvath, G. (2010). Artificial Neural Network based Local Motion Planning of a Wheeled Mobile Robot. International Symposium on Computational Intelligence and Informatics (CINTI) (pp. 213-218). Hungary: IEEE.

[12.] Miao, H., \& Tian, Y. (2013). Dynamic Robot Path Planning using an Enhanced Simulated Annealing Approach. ELSEVIER Applied Mathematics and Computation, 420-437.

[13.] Patle, B. K., Dayal, P., Jagadeesh, A., \& Sahu, O. (2017). Real Time Navigation Approach for Mobile Robot. Journal of Computers, $135-142$.

[14.] Alakshendra, V., \& Chiddarwar, S. (2016). Adaptive robust control of Mecanum-wheeled mobile robot with uncertainties. Nonlinear Dynamics. Nonlinear Dynamics, 1-23.

[15.] Alakshendra, V., Chiddarwar, S. S., Jha, A., \& Shaoo, S. (2017). Robust Trajectory Tracking Control for an Omnidirectional Mobile Robot. AIR doi:https://doi.org/10.1145/3132446.3134884.

[16.] Ammar, H., \& Azar, A. (2019). Robust Path Tracking of Mobile Robot Using Fractional Order PID Controller. In A. E. (Eds.), AMLTA 2019, AISC 921 (pp. 370-381). Springer Nature Switzerland AG 2020: Springer.

[17.] Sheth, S., Ajmera, A., Sharma, A., Patel, S., \& Kathrecha, C. (2018). Design and Development of Intelligent AGV Using Computer Vision and Artificial Intelligence. Soft Computing: Theories and Applications, Advances in Intelligent Systems and
Computing 583 , 337 - 349. doi:https://doi.org/10.1007/978-981-105687-1_31

[18.] Culler, D., \& Long, J. (2016). A Prototype Smart Materials Warehouse Application Implemented using Custom Mobile Robots and Open Source Vision Technology Developed using EmguCV. Procedia Manufacturing 44th Proceedings of the North American Manufacturing Research Institution of SME http://www.sme.org/namrc (pp. 1092 - 1106). Elsevier B.V.

[19.] Ito, S., Hiratsuka, S., Ohta, M., Matsubara, H., \& Ogawa, M. (2018). Small Imaging Depth LIDAR and DCNN-Based Localization for Automated Guided Vehicle. Sensors MDPI, 1-14.

[20.] Kala, R., Shukla, A., \& Tiwari, R. (2010). Dynamic Environment Robot Path Planning using Hierarchical Evolutionary Algorithms. Cybernetics and Systems, 435-454. doi:doi/abs/10.1080/01969722.2010.500800

[21.] Tazir, M. L., Azouaoui, O., Hazerchi, M., \& Brahimi, M. (2015). Mobile Robot Path Planning for Complex Dynamic Environment. IEEE.

[22.] Toolika, A., Yogita, G., \& Vijay, A. (2014). Robotic Path Planning using Genetic Algorithm in Dynamic Environment. International Journal of Computer Application.

[23.] Silva-Ortigoza, R., Márquez-Sánchez, C., Carrizosa-Corral, F., Hernández-Guzmán, V., García-Sánchez, J., Taud, H., . . . Álvarez-Cedillo, J. (2014). Obstacle Avoidance Task for a Wheeled Mobile Robot - A Matlab-Simulink-Based Didactic Application. In MATLAB Applications for the Practical Engineer (pp. 80-100). InTech. doi:http://dx.doi.org/10.5772/58392

[24.] Wu, Z., \& Feng, L. (2012). Obstacle Prediction-based Dynamic Path Planning for a Mobile Robot. International Journal of Advancements in Computing Technology(IJACT), IV(3), 118-124. doi:10.4156/ijact.vol4.issue3.16

[25.] Raja, P., \& Pugazhenthi, S. (2011). Path planning for a mobile robot in dynamic Environments. International Journal of the Physical Sciences, VI(20), 4721-4731.

[26.] El Khaili, M. (2014). Path Planning in a Dynamic Environment by the approach of the sliding on edge. International Journal of Advanced Computer Science and Applications (IJACSA), 86-92.

[27.] MandaI, P., Kumar, R., Maitra, M., \& Roy, S. (2013). Path Planning of Autonomous Mobile Robot: A New Approach. Proceedings of $7 \mathrm{~h}$ International Conference on Intelligent Systems and Control (ISCO 2013). IEEE.

[28.] Yun, S. C., Parasuraman, S., \& Ganapathy, V. (2011). Dynamic Path Planning Algorithm in Mobile Robot Navigation. Symposium on Industrial Electronics and Applications (ISIEA2011) (pp. 364-369). Langkawi, Malaysia: IEEE.

[29.] Li, W., Yang, C., Jiang, Y., Liu, X., \& Su, C.-Y. (2017). Motion Planning for Omnidirectional Wheeled Mobile Robot by Potential Field Method. Hindawi Journal of Advanced Transportation, 1-12.

[30.] Xu, Q.-L., Yu, T., \& Bai, J. (2017). The Mobile Robot Path Planning with Motion Constraints Based on Bug Algorithm. (pp. 2348-2352). IEEE.

[31.] Ayomoh, M., Omotuyi, O., Roux, A., \& Olufayo, O. (2018). Robot Navigation Model In a Multi-Target Domain Amidst Static and Dynamic Obstacles. Proceedings of the IASTED International Conference Intelligent Systems and Control (ISC 2018), (pp. 44-51). Calgary, Canada. doi:10.2316/P.2018.858-015

[32.] Hmeyda, F., \& Bouani, F. (2017). Camera-based autonomous Mobile Robot Path Planning and Trajectory tracking using PSO algorithm and PID Controller. ICCAD'17, Hammamet, January 1921 (pp. 203-208). Tunisia: IEEE. 
[33.] Xiaoyun, L., Qingrui, Z., Hailin, R., \& Changhao, S. (2018). Reinforcement Learning for Robot Navigation in Nondeterministic Environments. Proceedings of CCIS2018 (pp. 615-619). China: IEEE.

[34.] Ren, L., Wang, W., \& Du, Z. (2012). A New Fuzzy Intelligent Obstacle Avoidance Control Strategy for Wheeled Mobile Robot. International Conference on Mechatronics and Automation (ICMA), (pp. 1732-1737). China: IEEE.

[35.] Farooq, U., Hasan, K., Abbas, G., \& Asad, M. (2011). Comparative Analysis of Zero Order Sugeno and Mamdani Fuzzy Logic Controllers for Obstacle Avoidance Behavior in Mobile Robot Navigation. International Workshop on Current Trends in Information Technology (CTIT) (pp. 113-119). IEEE.

[36.] Muthu, T., Thierry, G., \& Swaminathan, S. S. (2012). Fuzzy Logic Controller for Autonomous Navigation. International Conference on Communications and Signal Processing (ICCSP) (pp. 81-92). India: IEEE.

[37.] Abdessemed, F., Faisal, M., \& Hedjar, R. (2014). A Hierarchical Fuzzy Control Design for Indoor Mobile Robot. International journal of Advance Robotic System, 11:33 I DOI: $10.5772 / 574434$.

[38.] Algabri, M., Mathkour, H., Ramdane, H., \& Alsulaiman, M. (2015). Comparative Study of Soft Computing Techniques for Mobile Robot Navigation in an Unknown Environment. Computers in Human Behavior, 42-56.

[39.] Mahmud, F., Arafat, A., \& Zuhori, S. (2012). Intelligent Autonomous Vehicle Navigated by Using Artificial Neural Network. International Conference on Electrical and Computer Engineering (pp. 105-108). Bangladesh: IEEE.

[40.] Ahmadzadeh, S., \& Ghanavati, M. (2012). Navigation of Mobile Robot Using the PSO Particle Swarm Optimization. Journal of Academic and Applied Studies (JAAS) 2(1), 32-38.

[41.] Castillo, O., Martinez-Marroquin, R., Melin, P., Valdez, F., \& Soria, J. (2012). Comparative Study of Bio-Inspired Algorithms Applied to the Optimization of Type-1 and Type-2 Fuzzy Controllers for an Autonomous Mobile Robot. ELSEVIER Information sciences 192: 19-38.

[42.] Zhang, Y., Gong, D., \& Zhang, J. (2013). Robot Path Planning in Uncertain Environment using Multi-Objective Particle Swarm Optimization. ELSEVIER Neurocomputing 103: , 172-185.

[43.] Shiltagh, N., \& Jalal, L. (2013). Optimal Path Planning for Intelligent Mobile Robot Navigation using Modified Particle Swarm Optimization. International Journal of Engineering and Advanced Technology 2(4):, 260-267.

[44.] Chung, H., Hou, C., \& Liu, S. (2013). Automatic Navigation of a Wheeled Mobile Robot using Particle Swarm Optimization and Fuzzy Control. International Symposium on Industrial Electronics (ISIE) (pp. 1-6). Taiwan: IEEE.

[45.] Juang, C., \& Chang, Y. (2011). Evolutionary-Group-based Particle Swarm Optimized Fuzzy Controller with Application to Mobile-Robot Navigation in Unknown Environments. Transactions on Fuzzy Systems. IEEE.

[46.] Allawi, Z., \& Abdalla, T. (2014). PSO-Optimized Type-2 Fuzzy Logic Controller for Navigation of Multiple Mobile Robots. International Conference on Methods and Models in Automation and Robotics (MMAR) (pp. 33-39). Poland: IEEE.

[47.] Dongshu, W., Yusheng, Z., \& Wenjie, S. (2011). BehaviorBased Hierarchical Fuzzy Control for Mobile Robot Navigation in Dynamic Environment. International Conference on Control and Decision Conference (CCDC) (pp. 2419-2424). China: IEEE.

[48.] Ayari, E., Hadouaj, S., \& Ghedira, K. (2010). A Fuzzy Logic Method for Autonomous Robot Navigation in Dynamic and Uncertain Environment Composed with Complex Traps. 5th International Multi-conference on Computing in the Global Information Technology (pp. 18-23). Spain: IEEE.

[49.] Nichols, E., McDaid, L., \& Siddique, N. (2013). Biologically Inspired SNN for Robot Control. Trans Cybern 15(2) (pp. 115-128). IEEE.

[50.] Al-Jarrah, R., Shahzad, A., \& Roth, H. (2015). Path Planning and Motion Coordination for Multi-Robots System Using Probabilistic Neuro Fuzzy. ELSEVIER IFAC-Papers Online 48(10), 46-51.

[51.] Kim, C., \& Chwa, D. (2015). Obstacle Avoidance Method for Wheeled Mobile Robots Using Interval Type-2 Fuzzy Neural Network. Transactions on Fuzzy Systems 23(3) (pp. 677-687). IEEE.

[52.] Brahmi, H., Ammar, B., \& Alimi, A. (2013). Intelligent Path Planning Algorithm for Autonomous Robot Based on Recurrent Neural Networks. International Conference on Advanced Logistics and Transport (ICALT) (pp. 199-204). Tunisia: IEEE.
[53.] Zhao, T., \& Wang, Y. (2012). Neural-Network based Autonomous Navigation System using Mobile Robots. 12th International Conference on Control, Automation, Robotics \& Vision (ICARCV) (pp. 1101-1106). China: IEEE.

[54.] Kumar, D., \& Dhama, K. (2012). Neuro-Fuzzy Control of an Intelligent Mobile Robot. 2nd International Conference on Advanced Computing \& Communication Technologies (ACCT) (pp. 106-111). India: IEEE.

[55.] Pothal, J., \& Parhi, D. (2015). Navigation of Multiple Mobile Robots in a Highly Clutter Terrains using Adaptive Neuro-Fuzzy Inference System. ELSEVIER Robotics and Autonomous Systems (72), 48-58.

[56.] Mohanta, J., Parhi, D., \& Patel, S. (2011). Path Planning Strategy for Autonomous Mobile Robot Navigation Using Petri-GA Optimization. Computers and Electrical Engineering 37(6): , 10581070.

[57.] Tuncer, A., \& Yildirim, M. (2012). Dynamic Path Planning of Mobile Robots with Improved Genetic Algorithm. ELSEVIER Computers and Electrical Engineering 38(6): 1564-1572.

[58.] Arora, T., Gigras, Y., \& Arora, V. (2014). Robotic Path Planning using Genetic Algorithm in Dynamic Environment. International Journal of Computer Application 89(11): 8-12.

[59.] Hussein, A., Mostafa, H., Badrel-din, M., Sultan, O., \& Khamis, A. (2012). Metaheuristic Optimization Approach to Mobile Robot Path Planning. International Conference on Engineering and Technology (ICET) (pp. 1-6). Egypt: IEEE.

[60.] Zhang, Q., Ma, J., \& Liu, Q. (2012). Path Planning based Quad tree Representation for Mobile Robot Using Hybrid-Simulated Annealing and Ant Colony Optimization Algorithm. International World Congress on Intelligent Control and Automation (pp. 25372542). China: IEEE.

[61.] Synodinos, A., \& Aspragathos, N. (2010). Robot Path Planning of a Mobile Robot using Solid Modeling Techniques on Potential Fields. International Conference on Mechatronics and Embedded Systems and Applications (MESA) (pp. 549-553). China: IEEE.

[62.] Algabri, M., Mathkour, H., \& Ramdane, H. (2014). Mobile Robot Navigation and Obstacle-Avoidance Using ANFIS in Unknown Environment. International Journal of Computer Applications 91(14), 36-41.

[63.] Singha, A., Ray, A. K., \& Samaddar, A. B. (2017). Navigation of Mobile Robot in a Grid-based Environment using Local and Target Weighted Environment using Local and Target Weighted. 8th ICCCNT IIT. Delhi: IEEE.

[64.] Hossain, M., \& Ferdousand, I. (2015). Autonomous Robot Path Planning in Dynamic Environment using a New Optimization Technique Inspired by Bacterial Foraging Technique. Robotics and Autonomous Systems 64: 137-141.

[65.] Liang, X., Li, L., Wu, J., \& Chen, H. (2013). Mobile Robot Path Planning based on Adaptive Bacterial Foraging Algorithm. Journal of Central South University 20(12), 3391-3400.

[66.] Liang, J.-H., \& Lee, C.-H. (2015). Efficient collision-free path-planning of multiple mobile robots' system. Advances in Engineering Software, 47-56. Retrieved from http://dx.doi.org/10.1016/j.advengsoft.2014.09.006

[67.] Brand, M., \& Yu, X. (2013). Autonomous Robot Path Optimization using Firefly Algorithm. International Conference on Machine Learning and Cybernetics (ICMLC) (pp. 1028-1032.). China: IEEE.

[68.] Mohajer, B., Kiani, K., Samiei, E., \& Sharifi, M. (2013). A New Online Random Particles Optimization Algorithm for Mobile Robot Path Planning in Dynamic Environments. Hindawi Mathematical Problems in Engineering, 1-9.

[69.] Purian, F., \& Sadeghian, E. (2013). Mobile Robots Path Planning using Ant Colony Optimization and Fuzzy Logic Algorithms in Unknown Dynamic Environments. International Conference on Control, Automation, Robotics and Embedded Systems (CARE) (pp. 1-6). India: IEEE.

[70.] Ganapathy, V., Jie, T., \& Parasuraman, S. (2010). Improved Ant Colony Optimization for Robot Navigation. International Symposium on Mechatronics and its Applications (ISMA) (pp. 1-6). United Arab Emirates: IEEE.

[71.] Hsu, C., Hou, R., \& Wang, W. (2013). Path Planning for Mobile Robots Based on Improved Ant Colony Optimization. International Conference on Systems, Man, and Cybernetics (SMC) (pp. 2777 - 2782). UK: IEEE.

[72.] Ganganath, N., Cheng, C., \& Tse, C. (2014). An ACO-based Off-line Path Planner for Nonholonomic Mobile Robots. 
International Symposium on Circuits and Systems (ISCAS) (pp. 1038-1041). Australia: IEEE.

[73.] Juang, C., Lai, M., \& Zeng, W. (2015). Evolutionary Fuzzy Control and Navigation for Two Wheeled Robots Cooperatively Carrying an Object in Unknown Environments. Transactions on Cybernetics 45(9) (pp. 1731-1743). IEEE.

[74.] Rusu, C., Birou, I., \& Szoke, E. (2010). Fuzzy Based Obstacle Avoidance System for Autonomous Mobile Robot. International Conference on Automation Quality and Testing Robotics (AQTR) (pp. 1-6). Romania: IEEE.

[75.] Masehian, E., \& Sedighizadeh, D. (2010). A Multi-Objective PSO-based Algorithm for Robot Path Planning. International Conference on Industrial Technology (ICIT) (pp. 465-470). Chile: IEEE.

[76.] Li, Q., Tang, Y., Wang, L., Zhang, C., \& Yin, Y. (2010). A Specialized Particle Swarm Optimization for Global Path Planning of Mobile Robots. 3rd International Workshop on Advanced Computational Intelligence (IWACI), (pp. 271-276). China: IEEE.

[77.] Chen, X., Kong, Y., Fang, X., \& Wu, Q. (2013). A Fast TwoStage ACO Algorithm for Robotic Path Planning. Neural Computing and Applications 22(2): 313-319.

[78.] Guillermo, E., Sunhong, P., \& Shuji, H. (2014). Contextual awareness in a WSN/RFID fusion navigation system. Hindawi Publishing Corporation, ISRN Robotics.

[79.] Arulselvi, S. (2014). Robot navigation System with RFID and Ultrasonic sensor. Middle-East Journal of Scientific Research, 11331137.

[80.] Shalev-Shwartz, S., \& Ben-David, S. (2014). Understanding Machine Learning from Theory to Algorithms. New York, NY, USA: 10013-2473.

[81.] Hu, J., \& Zhu, Q.-B. (2010). Path planning of robot for unknown environment based on prior knowledge rolling Q-learning. Control and Decision, Vol.25, No.9, 1364-1368.

[82.] Lakhmi, C. J., \& Atulya, K. N. (2013). A Deterministic Improved Q-Learning for Path Planning of a Mobile Robot. IEEE TRANSACTIONS ON SYSTEMS, MAN, AND CYBERNETICS: SYSTEMS, VOL. 43, NO. 5 (pp. 1141-1153). IEEE.

[83.] Ren, H., Yin, R., Li, F., Wang, W., \& Huo, M. (2016). Research on Q-ELM Algorithm in Robot Path Planning. 2016 28th Chinese Control and Decision Conference (CCDC) (pp. 5975-5979). IEEE.

[84.] Busoniu, L., Babuska, R., Schutter, B., \& Ernst, D. (2010). Reinforcement Learning and Dynamic Programming Using Function Approximators. Boca Raton, FL: CRC Press.

[85.] Yang, S., \& Li, C. (2017). Behavior Control Algorithm for Mobile Robot Based on Q- Learning. 2017 International Conference on Computer Network, Electronic and Automation (pp. 45-48). IEEE.

[86.] Ribeiro, T., Gonçalves, F., Garcia, I., Lopes, G., \& Ribeiro, A. (2019). Q-Learning for Autonomous Mobile Robot Obstacle Avoidance. IEEE.

[87.] Muhammad, J., \& Bucak, I. (2013). An improved Q-learning algorithm for an autonomous mobile robot navigation problem. (pp. 239-243). IEEE.

[88.] Watchanupaporn, O., \& Pudtuan, P. (2016). Multi-robot Target Reaching Using Modified Q-Iearning and PSO. 2016 The 2nd International Conference on Control, Automation and Robotics (pp. 66-69). IEEE.

[89.] Valiollahi, S., Ghaderi, R., \& Ebrahimzadeh, A. (2012). A Fuzzy Q-Learning Approach to Navigation of an Autonomous Robot. The 16th CSI International Symposium on Artificial Intelligence and Signal Processing (AISP 2012) (pp. 520-525). IEEE.

[90.] Xu, K., Wuy, F., \& Zhaoz, J. (2015). Simplified Online QLearning for LEGO EV3 Robot. 2015 IEEE International Conference on Control System, Computing and Engineering, 27 - 29 November (pp. 77-80). Penang, Malaysia: IEEE.

[91.] Zheng, Y., Wei, X., \& Zhou, X. (2019). Dynamic Obstacle Avoidance based on Multi-sensor Fusion and Q-Learning. 2019 IEEE 3rd Information Technology, Networking, Electronic and Automation Control Conference (ITNEC 2019) (pp. 1569-1573). IEEE.

[92.] Sichkar, V. N. (2019). Reinforcement Learning Algorithms in Global Path Planning for Mobile Robot. 2019 International Conference on Industrial Engineering, Applications and Manufacturing (ICIEAM) (pp. 1-5). IEEE.

[93.] Jiang, J., \& Xin, J. (2018). Path planning of a mobile robot in a free-space environment using Q-learning. Progress in Artificial Intelligence, 1-10.
[94.]

Li, S., Xu, X., \& Zuo, L. (2015). Dynamic Path Planning of a Mobile Robot with Improved Q-Learning algorithm. Proceeding of the 2015 IEEE International Conference on Information and Automation (pp. 409 - 414). Lijiang, China: IEEE.

[95.] Motlagh, O., Nakhaeinia, D., Tang, S., Karasfi, B., \& Khaksar, W. (2014). Automatic Navigation of Mobile Robots in Unknown Environments. Neural Computing and Applications 24(7), 1569-1581.

[96.] Roy, N., Chattopadhay, R., Mukherjee, A., \& Bhuiya, A. (2017). Implementation of Image Processing and Reinforcement Learning in Path Planning of Mobile Robots. International Journal of Engineering Science and Computing, 15211-15213.

[97.] Egerstedt, M. (2013, February 13). mouhknowsbest. Retrieved October 30, 2019, from youtube: https://youtu.be/aE7RQNhwnPQ

[98.] Rai, N., \& Rai, B. (2013). Neural Network based Closed loop Speed Control of DC Motor using Arduino Uno. International Journal of Engineering Trends and Technology, IV (2), 137-140.

[99.] Rossomando, F., \& Soria, C. (2015). Design and Implementation of Adaptive Neural PID for Non-Linear Dynamics in Mobile Robots. Latin America Transactions 13(4) (pp. 913-918). IEEE.

[100.] Al Mutib, K., \& Mattar, E. (2011). Neuro-fuzzy Controlled Autonomous Mobile Robotics System. 13th International Conference on Modelling and Simulation (pp. 1-7). UK: IEEE.

[101.] Baturone, I., Gersnoviez, A., \& Barriga, A. (2014). NeuroFuzzy Techniques to Optimize an FPGA Embedded Controller for Robot Navigation. ELSEVIER Applied Soft Computing 21: 95-106.

[102.] Deshpande, S., \& Bhosale, S. (2013). Adaptive Neuro-Fuzzy Inference System based Robotic Navigation. International Conference on Computational Intelligence and Computing Research (ICCIC) (pp. 1-4). IEEE.

[103.] Al-Mayyahi, A., Wang, W., \& Birch, P. (2014). Adaptive Neuro-Fuzzy Technique for Autonomous Ground Vehicle Navigation. Robotics, III (4), 349-370.

[104.] Jayaprakash, A., Bairampalli, S., Desai, V., \& Bhat, R. (2016). Development of Guided Autonomous Navigation for Indoor Material Handling Applications. Proceedings of SAI Intelligent Systems Conference (IntelliSys) 2016, Lecture Notes in Networks (pp. 584 - 595). Springer International Publishing AG 2018 Y. Bi et al. (eds.). doi:DOI 10.1007/978-3-319-56991-8_43

[105.] Lee, S. H., \& Chia, K. S. (2017). Navigating an Auto Guided Vehicle using Rotary Encoders and Proportional Controller. International Journal of Integrated Engineering, 71-77.

[106.] Koubaà, Y., Boukattaya, M., \& Damak, T. (2016). Path tracking control of non-holonomic wheeled mobile robot with skidding and slipping. International Journal of Modelling Identification and Control, 218-255.

[107.] Arantes, C., \& Sena Esteves, J. (2019). Omnidirectional Mobile Robot Platform with Four Mecanum Wheels Featuring Remote Motion Control Through Either a Graphical Application or an Inertial Measurement Unit. J. Machado et al. (Eds.): HELIX 2018, LNEE 505, 32-38, Retrieved from https://doi.org/10.1007/978-3319-91334-6 5

[108.] Susan, P., Young-Jae, R., \& Dae-Yeong, M. (2016). Fuzzy Steering Control of Three-Wheels Based Omnidirectional Mobile Robot. 1-6.

[109.] Ren, C., Li, X., Yang, X., \& Shugen, M. (2019). Extended State Observer based Sliding Mode Control of an Omnidirectional Mobile Robot with Friction Compensation. Transactions On Industrial Electronics (pp. 1-10). IEEE.

[110.] Mousavi, M. (2015). A Fast-Fuzzy Path Tracking Controller for Mobile Robots. Proceedings of the 3rd RSI International Conference on Robotics and Mechatronics (pp. 675-680). Tehran, Iran: IEEE.

[111.] Mahgoub, W., \& Sanhoury, R. (2017). Back Stepping Tracking controller for Wheeled Mobile Robot. International Conference on Communication, Control, Computing and Electronics Engineering (ICCCCEE) (pp. 1-5). Khartoum, Sudan: IEEE.

[112.] Alouache, A., \& Wu, Q. (2018). Genetic Algorithms for Trajectory Tracking of Mobile Robot Based on PID Controller. (pp. 237-241). IEEE.

[113.] Esmaeili, N., Alfi, A., \& Khosravi, H. (2017). Balancing and Trajectory Tracking of Two-Wheeled Mobile Robot Using Backstepping Sliding Mode Control: Design and Experiments. J Intell Robot Syst, 1-13. doi:10.1007/s10846-017-0486-9

[114.] Mallem, A., Nourredine, S., \& Benaziz, W. (2016). Mobile Robot Trajectory Tracking Using PID Fast Terminal Sliding Mode Inverse Dynamic Control. Proceedings of 2016 4th International 
Oluwaseun. O. Martins, Adefemi. A. Adekunle, Samuel. B. Adejuyigbe, Oluwole. H. Adeyemi and Michael. O. Arowolo/

Journal of Engineering Science and Technology Review 13 (3) (2020) 152 - 164

Conference on Control Engineering \& Information Technology (CEIT-2016) (pp. 1-6). Hammamet, Tunisia: IEEE.

[115.] Allagui, N., Ben Halima Abid, D., \& Derbel, N. (2018). Fuzzy PI controller for mobile robot navigation and tracking. 15th International Multi-Conference on Systems, Signals \& Devices (SSD) (pp. 1178-1183). IEEE.

[116.] Urrea, C., \& Muñoz, J. (2013). Path Tracking of Mobile Robot in Crops Performance Evaluations of Position Control. Journal of Intellegent Robot System. doi:DOI 10.1007/s10846-013-9989-1

[117.] Yousfi Allagui, N., Ben Halima Abid, D., \& Derbel, N. (2018). Fuzzy PI controller for mobile robot navigation and tracking. 2018. 15th International Multi-Conference on Systems, Signals \& Devices (SSD) (pp. 1178-1183). IEEE.

[118.] Dwivedi, S., Meena, R., Meena, R., Upadhyay, R., \& Padhy, P. (2014). Tracking of Mobile Robot using Hybrid Controller. International Conference on Advances in Engineering \&Technology Research (ICAETR) Dr Virendra Swarup Group of Institutions. Unnao, India: IEEE.

[119.] Lee, C.-T., Su, B.-R., Chang, C.-H., Hsu, T.-Y., \& Lee, W.D. (2018). Applications of Taguchi method to PID control for path tracking of a wheeled mobile robot. Proceedings of IEEE International Conference on Applied System Innovation IEEE ICASI 2018. IEEE.

[120.] Song, B., Zhang, Y., Cheng, J., \& Wang, J. (2010). Path Following Control of a Mobile Robot via Line-of Sight Method. Second International Conference on Intelligent Human-Machine Systems and Cybernetics (pp. 143-146). IEEE.
[121.] Heikkinen, J., Stotckaia, A., \& Minav, T. (2017). Self-tuning Parameter Fuzzy PID Controller for Autonomous Differential Drive Mobile Robot. (pp. 382-385). IEEE.

[122.] Zhi, W., Luo, Q.-S., \& Liu, J.-F. (2012). An Improved PID Tuning Algorithm for Mobile Robots. In D. J. (Eds.), Advances in ECWAC, Vol. 1, AISC 148 (pp. 345-353). Springer-Verlag Berlin Heidelberg 2012: springerlink.com.

[123.] Chang, H., \& Jin, T. (2013). Adaptive Tracking Controller Based on the PID for Mobile Robot Path Tracking. In J. L. (Eds.), ICIRA 2013, Part I, LNAI 8102 (pp. 540-549). Springer-Verlag Berlin Heidelberg: Springer.

[124.] Ammar, H., \& Azar, A. (2019). Robust Path Tracking of Mobile Robot Using Fractional Order PID Controller. In A. E. (Eds.), AMLTA 2019, AISC 921 (pp. 370-381). Springer Nature Switzerland AG 2020: Springer.

[125.] Tamila, S., Thiery, G., Shivashankar, S., \& Satish Kumar, L. (2012). Fuzzy logic controller for autonomous Navigation. IEEE.

[126.] Salem, F. (2013). Kinematics and Dynamic Models and Control for Differential Drive Mobile. International Journal of Current Engineering and Technology, 253-263.

[127.] Barthelmes, S., \& Zehnter, S. (2017). An All-TerrainController for Over-Actuated Wheeled Mobile Robots with Feedforward and Optimization-Based Control Allocation. 56th Annual Conference on Decision and Control (CDC) (pp. 52155222). Melbourne, Australia: IEEE.

[128.] Proaño, P., Capito, L., Rosales, A., \& Camacho, O. (2015). Sliding Mode Control: Implementation like PID for trajectorytracking for Mobile Robots. Asia-Pacific Conference on Computer Aided System Engineering (pp. 220-225). IEEE. 